\title{
Decay Constants of S Wave Heavy Quarkonia
}

\section{Bhaghyesh Azhothkaran ${ }^{1}\left[\right.$ ] $\cdot$ Nilakanthan V. K. ${ }^{2}$}

Received: 3 December 2019 / Accepted: 22 April 2020 / Published online: 9 May 2020

(C) The Author(s) 2020

\begin{abstract}
We derive expressions for the decay constants of $\mathrm{S}$ wave pseudoscalar and vector quarkonium $(Q \bar{Q})$ states through approximating the quark model definition by including terms upto $k^{2} / c^{2}$. Using the derived formula, the decays constants for ground and excited states are calculated. Our predictions are found to be in good agreement with the available experimental and lattice results.
\end{abstract}

Keywords Decay constants · Quarkonia · Potential model

\section{Introduction}

Ever since the discovery of first charmonium state $(J / \Psi)$ at SLAC and BNL, quarkonia spectroscopy has always been a subject of continuous research, both experimentally and theoretically $[1,2]$. Thanks to the experimental facilities at various colliders, new states have been constantly discovered [2,3]. Recently discovered conventional quarkonium states include $\eta_{b}(1 S), \eta_{b}(2 S), h_{b}(1 P), h_{b}(2 P), \chi_{b 1}(3 P)$ and $\chi_{b 2}(3 P)$ [3]. There are also lot of states that are discovered (named $\mathrm{X}, \mathrm{Y}, \mathrm{Z}$ ) that do not fit into the conventional quarkonia spectra and are assumed to be strong candidates for non-conventional hadronic states [26]. Comprehensive reviews on the status of heavy quarkonium physics can be found in References [5-13].

Even though quantum chromodynamics (QCD) is the theory of strong interactions, there is no direct method to extract the properties of observed hadrons directly from QCD Lagrangian. Lattice QCD provides great hope in this direction and significant results are being reported [1,2]. Various other approaches widely used in the literature to study hadronic systems include QCD sum rules [14-19], nonrelativistic and relativistic potential models [20-30, 32-36], effective field theory [37-39] and formalisms based on BetheSalpeter equation and Dyson-Schwinger equation [40-49]. Among theses QCD inspired

Bhaghyesh Azhothkaran

bhaghyesh.mit@manipal.edu

1 Department of Physics, Manipal Institute of Technology, Manipal Academy of Higher Education, 576 104, Manipal, India

2 Department of Post Graduate Studies and Research in Physics, St Aloysius College (Autonomous), 575 003, Mangalore, India 
potential models have been largely successful in explaining hadronic properties. Importance of potential models stems from the fact that masses (upto rather high excitations) and decay properties can be investigated in an unified way.

One of the important quantities that can be obtained through potential models are the vector and pseudoscalar decay constants of heavy mesons. Decay constants are important because it can be used to estimate the hadronic matrix elements and provide information about the short distance structure of hadrons and non-perturbative QCD dynamics [19, 27]. Precise knowledge of their values play very important roles, for example in determination of CKM matrix elements, study of weak decays, meson mixing, etc. $[44,50]$. They are input parameters which constrains various decays and processes and their precise knowledge is important in various experimental measurements [51, 52]. Vector $\left(f_{V}\right)$ and pseudoscalar $\left(f_{P}\right)$ decay constants are defined through the matrix elements [27]:

$$
\begin{aligned}
m_{V} f_{V} \epsilon^{\mu} & =\left\langle 0\left|\bar{\Psi} \gamma^{\mu} \Psi\right| V\right\rangle \\
p^{\mu} f_{P} & =i\left\langle 0\left|\bar{\Psi} \gamma^{\mu} \gamma^{5} \Psi\right| P\right\rangle
\end{aligned}
$$

The quark model definition of decay constants are through [25, 27]:

$$
\begin{aligned}
f_{V} & =\sqrt{\frac{3}{M}} \int \frac{d^{3} k}{(2 \pi)^{3}} \sqrt{1+\frac{m_{Q}}{E_{k}}} \sqrt{1+\frac{m_{\bar{Q}}}{E_{\bar{k}}}}\left(1+\frac{k^{2}}{3\left(E_{k}+m_{Q}\right)\left(E_{\bar{k}}+m_{\bar{Q}}\right)}\right) \phi(\mathbf{k}) \\
f_{P} & =\sqrt{\frac{3}{M}} \int \frac{d^{3} k}{(2 \pi)^{3}} \sqrt{1+\frac{m_{Q}}{E_{k}}} \sqrt{1+\frac{m_{\bar{Q}}}{E_{\bar{k}}}}\left(1-\frac{k^{2}}{\left(E_{k}+m_{Q}\right)\left(E_{\bar{k}}+m_{\bar{Q}}\right)}\right) \phi(\mathbf{k})
\end{aligned}
$$

The above expressions are mostly used in potential models which use the momentum space representation. In the non-relativistic limit $(k \rightarrow 0)$, one obtains the well-known Van Royen-Weisskopf formula which relates the decay constants to the value of the meson wave-function at the origin [53]:

$$
f_{P / V}^{2}=12 \frac{\left|\psi_{P / V}(0)\right|^{2}}{M_{P / V}}
$$

In (3), $\psi_{P / V}(0)$ is the wave function of the pseudoscalar/vector meson calculated at the ori$\operatorname{gin}(r=0)$ and $M_{P / V}$ is the mass of the pseudoscalar/vector meson. This formula is widely used in potential model calculations [22-25, 27, 29, 32-35]. One reason that it is widely used is that the coordinate space wave function is sufficient to calculate the decay constants. In potential models, in which the vector and pseudoscalar states differ only by the spindependent corrections, one obtains $f_{P} \approx f_{V}[22-25,32,35,54]$. The relativistic effects which enters as the $v^{2} / c^{2}$ corrections in hyperfine spltting cannot be neglected in quarkonia. They produce significant difference in the wavefunction at origin for pseudoscalar and vector mesons [55], and hence $f_{P}$ becomes very different from $f_{V}$. Ebert et al. [25] have shown that inclusion of relativistic effects produces considerable $(\sim 70 \mathrm{MeV})$ difference between vector and pseudoscalar decay constants. Also the present experimental estimates $\left(f_{J / \psi}=416 \pm 6 \mathrm{MeV}[3]\right.$ and $f_{\eta_{c}}=335 \pm 75 \mathrm{MeV}$ [56]), lattice [19, 52] and other theoretical studies $[19,44,45,48,50,57,58]$ predict that $f_{V}$ is greater than $f_{P}$. Hence the non-relativistic Van Royen-Weisskopf formula fails to provide exact results in accordance to experimental and lattice results. We suggest that the higher order terms $\left(\mathcal{O}\left(k^{2} / c^{2}\right)\right)$ in (1) and (2) are important. In this paper, we derive expressions for the decay constants of S-wave quarkonia by approximating the quark model definition by including terms upto $k^{2} / c^{2}$. 
This paper is organised as follows: in Section 2 we derive expressions for pseudoscalar and vector decay constants for S-wave quarkonia including the $k^{2} / c^{2}$ corrections. In Section 3 we discuss the theoretical model used in the present analysis. Results and discussions of our present analysis are given in Section 4.

\section{Decay Constants}

For quarkonia $m_{Q}=m_{\bar{Q}}=m$ and hence $E_{k}=E_{\bar{k}}=E$. Also using the relation, $k^{2}=$ $(E+m)(E-m),(1)$ becomes

$$
\begin{aligned}
f_{V} & =\sqrt{\frac{3}{M}} \int \frac{d^{3} k}{(2 \pi)^{3}}\left(1+\frac{m}{E}\right)\left(1+\frac{(E+m)(E-m)}{3(E+m)(E+m)}\right) \phi(\mathbf{k}) \\
& =\frac{1}{3} \sqrt{\frac{3}{M}} \int \frac{d^{3} k}{(2 \pi)^{3}}\left(4+\frac{2 m}{E}\right) \phi(\mathbf{k})
\end{aligned}
$$

Now,

$$
\begin{aligned}
E=\left(k^{2}+m^{2}\right)^{1 / 2} & =\left[m^{2}\left(1+\frac{k^{2}}{m^{2}}\right)\right]^{1 / 2} \\
& =m\left(1+\frac{k^{2}}{2 m^{2}}\right)+\mathcal{O}\left(\frac{k^{4}}{m^{4}}\right)
\end{aligned}
$$

Substituting (5) in (4), we get

$$
\begin{aligned}
f_{V} & =\frac{1}{3} \sqrt{\frac{3}{M}} \int \frac{d^{3} k}{(2 \pi)^{3}}\left(4+\frac{2 m}{m\left(1+\frac{k^{2}}{2 m^{2}}\right)}\right) \phi(\mathbf{k}) \\
& =\frac{1}{3} \sqrt{\frac{3}{M}} \int \frac{d^{3} k}{(2 \pi)^{3}}\left(4+\frac{4 m^{2}}{k^{2}+2 m^{2}}\right) \phi(\mathbf{k})
\end{aligned}
$$

Now using the three dimensional Fourier transform of $\phi(\mathbf{k})$ the second integral in (6) can be written as:

$$
\begin{aligned}
\int \frac{d^{3} k}{(2 \pi)^{3}}\left(\frac{4 m^{2}}{k^{2}+2 m^{2}}\right) \phi(\mathbf{k}) & =4 m^{2} \int \frac{d^{3} k}{(2 \pi)^{3}} \frac{1}{k^{2}+2 m^{2}} \int d^{3} r e^{-i \mathbf{k} \cdot \vec{r}} \tilde{\phi}(\vec{r}) \\
& =4 m^{2} \int d^{3} r \tilde{\phi}(\vec{r}) \int \frac{d^{3} k}{(2 \pi)^{3}} \frac{e^{-i \mathbf{k} \cdot \vec{r}}}{k^{2}+2 m^{2}} \\
& =4 m^{2} \int d^{3} r \tilde{\phi}(\vec{r}) \int \frac{d^{3} k}{(2 \pi)^{3}} \frac{e^{-i \mathbf{k} \cdot \vec{r}}}{k^{2}+(\sqrt{2} m)^{2}} \\
& =4 m^{2} \int d^{3} r \tilde{\phi}(\vec{r}) \cdot \frac{1}{4 \pi r} e^{-\sqrt{2} m r} \\
& =\frac{m^{2}}{\pi} \int d^{3} r \frac{\tilde{\phi}(\vec{r})}{r} e^{-\sqrt{2} m r}
\end{aligned}
$$

For S-wave states, there is no angular dependence:

$$
\tilde{\phi}(\vec{r})=\frac{R(r)}{\sqrt{4 \pi}}
$$


Therefore, (7) becomes:

$$
\begin{aligned}
\int \frac{d^{3} k}{(2 \pi)^{3}}\left(\frac{4 m^{2}}{k^{2}+2 m^{2}}\right) \phi(\mathbf{k}) & =\frac{m^{2}}{\pi} \frac{1}{\sqrt{4 \pi}} \int d^{3} r \frac{R(r)}{r} e^{-\sqrt{2} m r} \\
& =\frac{m^{2}}{\pi} \frac{1}{\sqrt{4 \pi}} 4 \pi \int d r \frac{R(r)}{r} r^{2} e^{-\sqrt{2} m r} \\
& =\sqrt{\frac{4}{\pi}} m^{2} \int d r R(r) r e^{-\sqrt{2} m r}
\end{aligned}
$$

Similarly the integral

$$
\int \frac{d^{3} k}{(2 \pi)^{3}} \phi(\mathbf{k})=\tilde{\phi}(\vec{r}=0)=\frac{R(0)}{\sqrt{4 \pi}}
$$

Substituting (9) and (10) in (6), we get the final expression for vector decay constant of S-wave quarkonia as:

$$
f_{V}=\frac{1}{3} \sqrt{\frac{3}{M}} \sqrt{\frac{4}{\pi}}\left[R(0)+m^{2} \int d r R(r) r e^{-\sqrt{2} m r}\right]
$$

Similarly setting $m_{Q}=m_{\bar{Q}}=m, E_{k}=E_{\bar{k}}=E$ and $k^{2}=(E+m)(E-m),(2)$ becomes

$$
\begin{aligned}
f_{P} & =\sqrt{\frac{3}{M}} \int \frac{d^{3} k}{(2 \pi)^{3}}\left(1+\frac{m}{E}\right)\left(1-\frac{(E+m)(E-m)}{(E+m)(E+m)}\right) \phi(\mathbf{k}) \\
& =\sqrt{\frac{3}{M}} \int \frac{d^{3} k}{(2 \pi)^{3}} \frac{2 m}{E} \phi(\mathbf{k})
\end{aligned}
$$

Substituting (5) in (12), we get:

$$
f_{P}=\sqrt{\frac{3}{M}} \int \frac{d^{3} k}{(2 \pi)^{3}} \frac{4 m^{2}}{k^{2}+2 m^{2}} \phi(\mathbf{k})
$$

Fourier tranforming $\phi(\mathbf{k})$ and performing the integrals as done earlier, we obtain the final expression for pseudoscalar decay constant of S-wave quarkonia as:

$$
f_{P}=\sqrt{\frac{3}{M}} \sqrt{\frac{4}{\pi}} m^{2} \int d r R(r) r e^{-\sqrt{2} m r}
$$

Taking into account the QCD corrections [59-61], the decay constants of vector and pseudoscalar states equal to

$$
\bar{f}_{V / P}=f_{V / P}\left(1-\delta_{V / P} \frac{\alpha_{S}}{\pi}\right)
$$

where $\delta_{V}=8 / 3$ and $\delta_{P}=2$.

\section{Theoretical Model}

In order to obtain the bound state wavefunctions of heavy mesons, we have used a screened potential model suggested by $\mathrm{Li}$ and Chao $[30,31]$. The quark-antiquark potential in this model is given by

$$
V(r)=-\frac{4}{3} \frac{\alpha_{C}}{r}+\lambda\left(\frac{1-e^{-\mu r}}{\mu}\right)+V_{0}
$$


The hyperfine interaction term which provide the spin singlet-triplet splitting is

$$
H_{h f}=\frac{32 \pi \alpha_{C}}{9 m_{Q}^{2}} \delta_{\sigma}(r) \vec{S}_{Q} \cdot \vec{S}_{\bar{Q}},
$$

where $\delta_{\sigma}(r)=(\sigma / \sqrt{\pi})^{3} e^{-\sigma^{2} r^{2}}$. The hyperfine interaction is treated perturbatively. The parameters used in (15) and (16) are same as in References [30, 31] and are listed in Table 1 for completeness.

Using the potential (15), we solve the corresponding non-relativistic Schrodinger equation numerically [62] and obtain the bound state wavefunctions for heavy mesons. Substituting the wavefunctions in (14) we obtain respectively the vector and pseudoscalar decay constants.

To check the validity of our numerical computation method, we calculate the mass spectra and the radial wavefunction at the origin $\left(|R(0)|^{2}\right)$ and compare the results with that of References [30, 31]. The results are presented in Tables 2 and 3 along with the spinaveraged masses $\left(M_{S A}\right)$ and hyperfine contributions. $\left\langle H_{h f}^{V}\right\rangle$ and $\left\langle H_{h f}^{P}\right\rangle$ are respectively the hyperfine contributions for spin-triplet (vector) and spin-singlet (pseudoscalar) states. The $|R(0)|^{2}$ of References $[30,31]$ are extracted from the corresponding leptonic decay widths. The mass spectra and $|R(0)|^{2}$ obtained in the present analysis are in good agreement with the corresponding results of References [30,31], confirming the validity of our numerical computation method.

\section{Results and Discussions}

The computed values of pseudoscalar and vector decay constants are presented in Tables 4 and 5. $f^{N R}$ and $\bar{f}^{N R}$ are respectively the decay constants calculated using the nonrelativistic Van Royen-Weisskopf formula (3) without and with the QCD correction factor. $f$ and $\bar{f}$ are respectively the decay constants calculated from our present analysis $((11)$ and (13)) without and with the QCD correction factor (14).

In Tables 6, 7, 8 and 9, pseudoscalar $\left(\bar{f}_{P}\right)$ and vector $\left(\bar{f}_{V}\right)$ decay constants for charmonium and bottomonium states obtained from our analysis are compared with the available experimental results [3, 56] and predictions from lattice [19, 52, 63-68], nonrelativistic potential models [27, 69], QCD sum rules (QCDSR) [19, 57, 58], light front quark model (LFQM) [50] and formalisms based on Bethe-Salpeter and Dyson-Schwinger equations (BS/DSE) [44-49].

Vector decay constants are experimentally extracted from the leptonic decay widths through the relation

$$
\Gamma\left(V \rightarrow e^{+} e^{-}\right)=\frac{4 \pi}{3} \alpha^{2} e_{Q}^{2} \frac{f_{V}^{2}}{M_{V}}
$$

Substituting the PDG averages for $\Gamma\left(V \rightarrow e^{+} e^{-}\right)$in the above expression, one can estimate the vector decay constants. As far as the pseudoscalar decay constants are concerned, only the $f_{n_{c}}$ is experimentally measured through the $B \rightarrow n_{c} K$ decay [56]. $f_{n_{b}}$ is not yet experimentally determined.

Table 1 Model Parameters

\begin{tabular}{lllllll}
\hline Meson & $\alpha_{C}$ & $\mu$ & $m_{Q}(\mathrm{GeV})$ & $\lambda\left(\mathrm{GeV}^{2}\right)$ & $\sigma(\mathrm{GeV})$ & $V_{0}(\mathrm{GeV})$ \\
\hline$c \bar{c}$ & 0.5007 & 0.0979 & 1.4045 & 0.21 & 1.362 & 0 \\
$b \bar{b}$ & 0.37 & 0.056 & 4.4 & 0.21 & 3.3 & 0.677 \\
\hline
\end{tabular}


Table 2 Charmonium spectrum (in $\mathrm{GeV}$ ) and radial wavefunctions at the origin (in $\mathrm{GeV}^{3}$ )

\begin{tabular}{lccccccccc}
\hline State & $M_{S A}$ & $\left\langle H_{h f}^{V}\right\rangle$ & $\left\langle H_{h f}^{P}\right\rangle$ & $M_{V}$ & $M_{P}$ & $M_{V}[30]$ & $M_{P}[30]$ & $|R(0)|^{2}$ & $|R(0)|^{2}[30]$ \\
\hline $1 S$ & 3.067 & 0.030 & -0.088 & 3.097 & 2.979 & 3.097 & 2.979 & 1.19 & 1.19 \\
$2 S$ & 3.661 & 0.012 & -0.037 & 3.673 & 3.624 & 3.673 & 3.623 & 0.61 & 0.61 \\
$3 S$ & 4.014 & 0.008 & -0.024 & 4.022 & 3.990 & 4.022 & 3.991 & 0.43 & 0.43 \\
$4 S$ & 4.267 & 0.006 & -0.017 & 4.273 & 4.250 & 4.273 & 4.250 & 0.33 & 0.33 \\
\hline
\end{tabular}

Table 3 Bottomonium spectrum (in $\mathrm{GeV}$ ) and radial wavefunctions at the origin (in $\mathrm{GeV}^{3}$ )

\begin{tabular}{llllllllll}
\hline State & $M_{S A}$ & $\left\langle H_{h f}^{V}\right\rangle$ & \multicolumn{1}{c}{$\left\langle H_{h f}^{P}\right\rangle$} & \multicolumn{1}{c}{$M_{V}$} & \multicolumn{1}{c}{$M_{P}$} & \multicolumn{1}{c}{$M_{V}[31]$} & \multicolumn{1}{c}{$M_{P}[31]$} & $|R(0)|^{2}$ & $|R(0)|^{2}[31]$ \\
\hline $1 S$ & 9.442 & 0.018 & -0.054 & 9.460 & 9.388 & 9.460 & 9.389 & 8.72 & 8.73 \\
$2 S$ & 10.009 & 0.007 & -0.021 & 10.016 & 9.988 & 10.016 & 9.987 & 3.88 & 3.89 \\
$3 S$ & 10.346 & 0.005 & -0.015 & 10.351 & 10.331 & 10.351 & 10.330 & 2.87 & 2.89 \\
$4 S$ & 10.607 & 0.004 & -0.012 & 10.611 & 10.596 & 10.611 & 10.595 & 2.39 & 2.43 \\
\hline
\end{tabular}

Table 4 Decay constants (in $\mathrm{MeV}$ ) of charmonium states in various schemes

Table 5 Decay constants (in $\mathrm{MeV}$ ) of bottomonium states in various schemes

\begin{tabular}{lllll}
\hline State & $f^{N R}$ & $\bar{f}^{N R}$ & $f$ & $\bar{f}$ \\
\hline$\eta_{c}(1 S)$ & 618 & 516 & 375 & 313 \\
$\eta_{c}(2 S)$ & 402 & 336 & 207 & 173 \\
$\eta_{c}(3 S)$ & 323 & 269 & 152 & 127 \\
$\eta_{c}(4 S)$ & 273 & 227 & 122 & 102 \\
$J / \psi$ & 607 & 473 & 527 & 411 \\
$\psi(2 S)$ & 400 & 311 & 335 & 261 \\
$\psi(3 S)$ & 321 & 250 & 265 & 206 \\
$\psi(4 S)$ & 272 & 212 & 222 & 173 \\
\hline
\end{tabular}

\begin{tabular}{lllll}
\hline State & $f^{N R}$ & $\bar{f}^{N R}$ & $f$ & $\bar{f}$ \\
\hline$\eta_{b}(1 S)$ & 940 & 833 & 671 & 594 \\
$\eta_{b}(2 S)$ & 608 & 538 & 410 & 363 \\
$\eta_{b}(3 S)$ & 515 & 456 & 336 & 298 \\
$\eta_{b}(4 S)$ & 463 & 410 & 296 & 262 \\
$\Upsilon(1 S)$ & 937 & 794 & 847 & 718 \\
$\Upsilon(2 S)$ & 607 & 515 & 541 & 459 \\
$\Upsilon(3 S)$ & 514 & 436 & 455 & 385 \\
$\Upsilon(4 S)$ & 463 & 392 & 407 & 345 \\
\hline
\end{tabular}




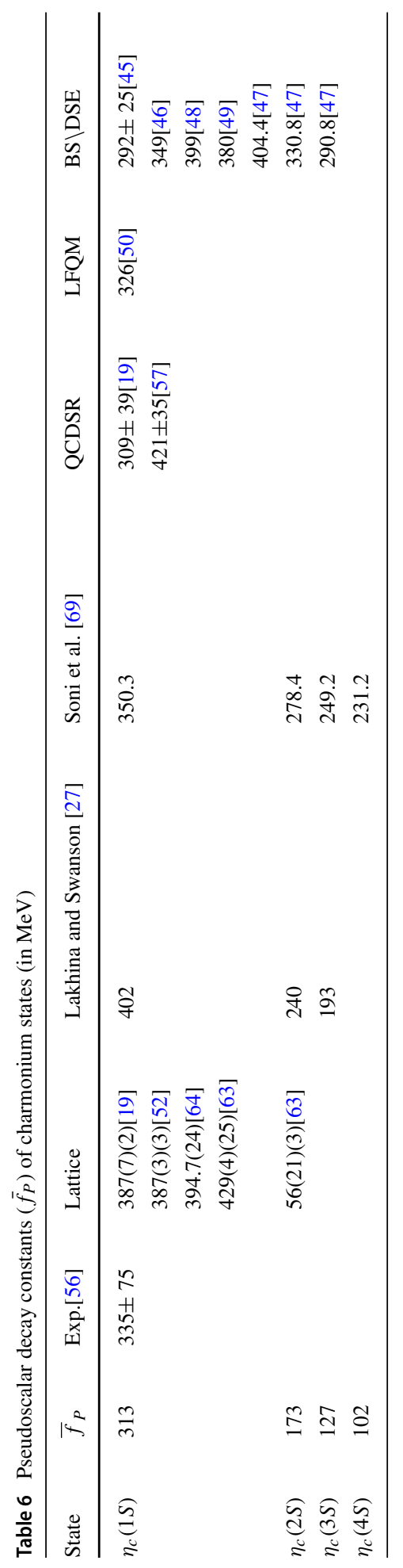




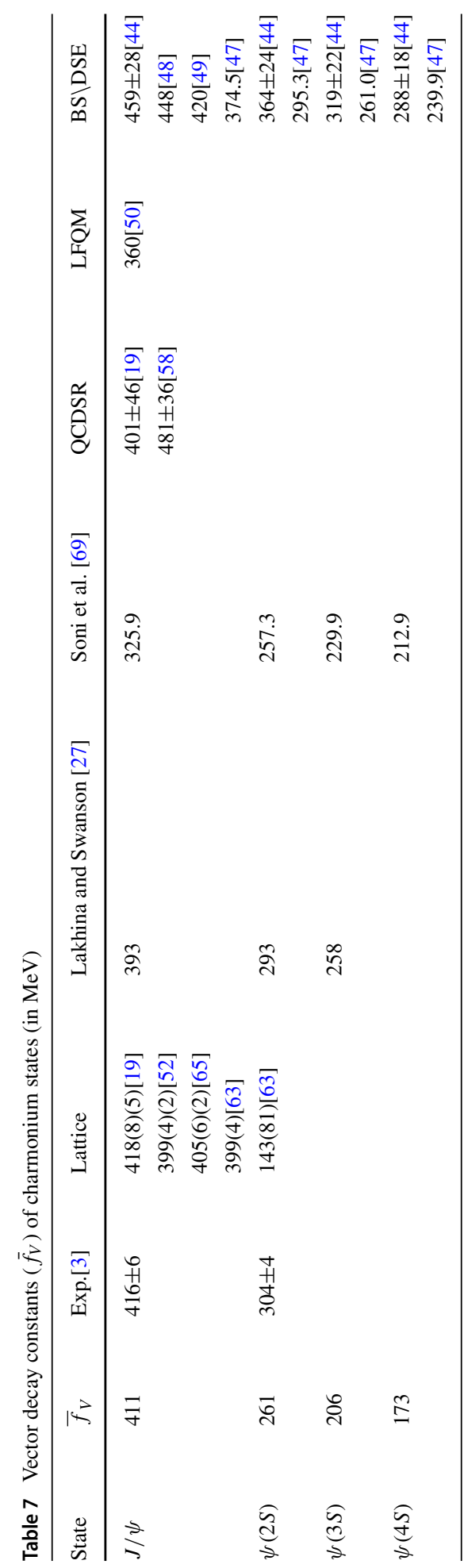




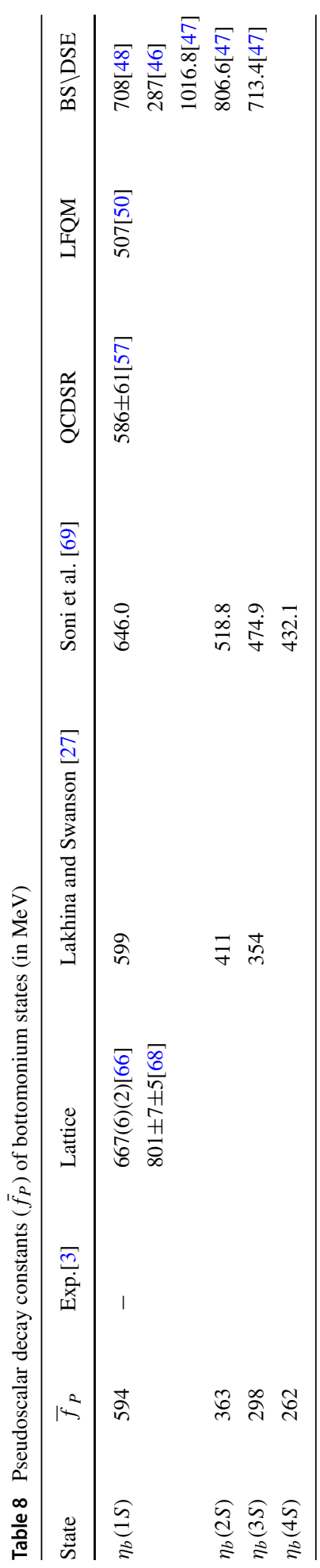




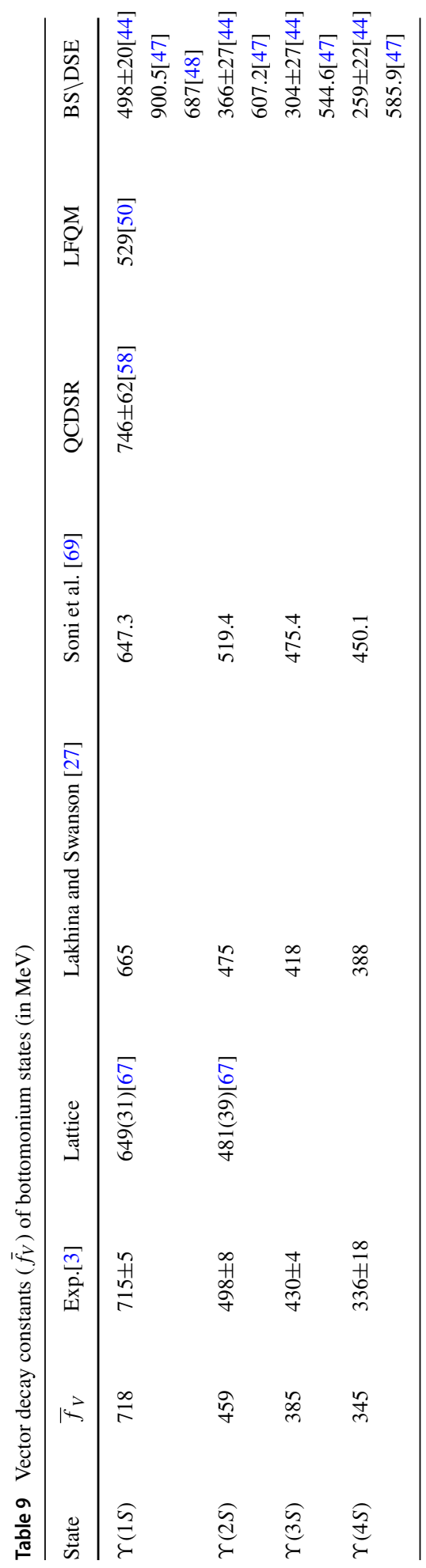




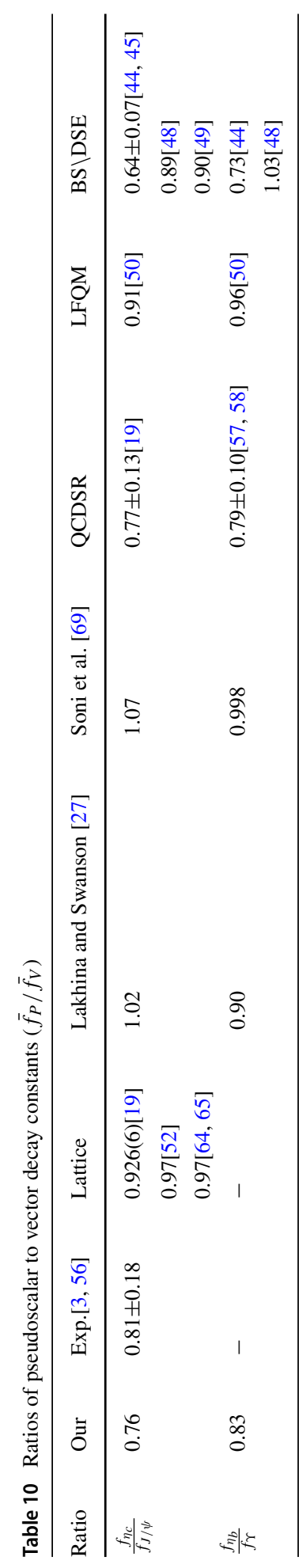


As seen from Tables 6-9, the predictions of decay constants from various models show a wide range of variation. For most of the states, the experimental results are yet to be available. Results from our analysis are found to be in good agreement with the available experimental results. For both vector and pseudoscalar states, the decay constants keep decreasing with the radial excitation number which is also in accordance with existing experimental/lattice results and other theoretical studies.

The ratios of pseudoscalar to vector decay constant $\left.\left(\bar{f}_{P} / \bar{f}_{V}\right)\right)$ for the $1 \mathrm{~S}$ states are shown in Table 10. For both $c \bar{c}$ and $b \bar{b}$ mesons, we obtain $\bar{f}_{P} / \bar{f}_{V}<1$, which is consistent with the available experimental data $\left(f_{\eta_{c}} / f_{J / \psi}=0.81 \pm 0.18[3,56]\right)$ and other theoretical predictions. This result is in sharp contrast with predictions of some of the nonrelativistic potential models $[22-25,32,35]$ which predict $f_{P} / f_{V} \approx 1$. This indicate that for quarkonia the relativistic effects are important in calculating the decay constants.

In summary, we have derived expressions for pseudoscalar and vector decay constants for S-wave quarkonia including the $k^{2} / c^{2}$ corrections. Our predictions are consistent with the experiment and predictions from other theoretical studies.

Acknowledgments One of the authors (Bhaghyesh) would like to thank Manipal Academy of Higher Education (MAHE), Manipal for covering the article processing charges for open access publication.

Funding Information Open access funding provided by Manipal Academy of Higher Education, Manipal.

Open Access This article is licensed under a Creative Commons Attribution 4.0 International License, which permits use, sharing, adaptation, distribution and reproduction in any medium or format, as long as you give appropriate credit to the original author(s) and the source, provide a link to the Creative Commons licence, and indicate if changes were made. The images or other third party material in this article are included in the article's Creative Commons licence, unless indicated otherwise in a credit line to the material. If material is not included in the article's Creative Commons licence and your intended use is not permitted by statutory regulation or exceeds the permitted use, you will need to obtain permission directly from the copyright holder. To view a copy of this licence, visit http://creativecommons.org/licenses/by/4.0/.

\section{References}

1. Bodwin, G.T., et al.: A Snowmass White Paper. arXiv:1307.7425v3

2. Brambilla, N., et al., (Quarkonium Working Group): Eur. Phys. J. C 71, 1534 (2011). arXiv:1010.5827v3

3. Patrignani, C., et al., (Particle Data Group): Chin. Phys. C 40, 100001 (2016)

4. Godfrey, S., Olsen, S.L.: Ann. Rev. Nucl. Part. Sci. 58, 51 (2008). arXiv:0801.3867v1

5. Olsen, S.L.: Front. Phys. (Beijing) 10, 121 (2015). arXiv:1411.7738

6. Lebed, R.F., Mitchell, R.E., Swanson, E.S.: Prog. Part. Nucl. Phys. 93, 143 (2017). arXiv:1610.04528

7. Esposito, A., et al.: Int. J. Mod. Phys. A 30, 1530002 (2015). arXiv:1411.5997

8. Chen, H.X., Chen, W., Liu, X., Zhu, S.L.: Phys. Rept. 639, 1 (2016). arXiv:1601.02092

9. Ali, A., Lange, J.S., Stone, S.: Prog. Part. Nucl. Phys. 97, 123 (2017). arXiv:1706.00610

10. Guo, F.K., et al.: Rev. Mod. Phys. 90, 015004 (2018). arXiv:1705.00141

11. Olsen, S.L., Skwarnicki, T., Zieminska, D.: Rev. Mod. Phys. 90, 015003 (2018). arXiv:1708.04012

12. Brambilla, N., et al.: Eur. Phys. J. C 71, 1534 (2011). arXiv: 1010.5827

13. Brambilla, N., et al.: Eur. Phys. J. C 74, 2981 (2014). arXiv:1404.3723

14. Shifman, M.A., Vainshtein, A.I., Zakharov, V.I.: Nucl. Phys. B 147, 385 (1979). Nucl. Phys. B 14, 448 (1979)

15. Reinders, L.J., Rubinstein, H.R., Yazaki, S.: Nucl. Phys. B 186, 109 (1981)

16. Novikov, V.A., Okun, L.B., Shifman, M.A., Vainshtein, A.I., Voloshin, M.B., Zakharov, V.I.: Phys. Rept. 41, 1 (1978)

17. Beilin, V.A., Radyushkin, A.V.: Nucl. Phys. B 260, 61 (1985)

18. Nielsen, M., Navarra, F.S., Lee, S.H.: Phys. Rept. 497, 41 (2010). arXiv:0911.1958v2

19. Becirevic, D., Duplancic, G., Klajn, B., Melic, B., Sanfilippo, F.: Nucl. Phys. B 883, 306 (2014). arXiv:1312.2858v3 
20. Eichten, E., Gottfried, K., Kinoshita, T., Kogut, J., Lane, K.D., Yan, T.M.: Phys. Rev. Lett. 34, 369 (1975)

21. Eichten, E., Gottfried, K., Kinoshita, T., Lane, K.D., Yan, T.M.: Phys. Rev. D 17, 3090 (1978). Erratum Phys. Rev. D 21, 313 (1980)

22. Eichten, E.J., Quigg, C.: Phys. Rev. D 49, 5845 (1994). arXiv:hep-ph/9402210v1

23. Gershtein, S.S., Kiselev, V.V., Likhoded, A.K., Tkabladze, A.V.: Phys. Rev. D 51, 3613 (1995). arXiv:hep-ph/9406339v1

24. Fulcher, L.P.: Phys. Rev. D 60, 074006 (1999). arXiv:hep-ph/9806444v2

25. Ebert, D., Faustov, R.N., Galkin, V.O.: Phys. Rev. D 67, 014027 (2003). arXiv:hep-ph/0210381v2

26. Barnes, T., Godfrey, S., Swanson, E.S.: Phys. Rev. D 72, 054026 (2005). arXiv:hep-ph/0505002v3

27. Lakhina, O., Swanson, E.S.: Phys. Rev. D 74, 014012 (2006). arXiv:hep-ph/0603164v2

28. Radford, S.F., Repko, W.W.: Phys. Rev. D 074031, 75 (2007). arXiv:hep-ph/0701117v3

29. Ikhdair, S.M., Sever, R.: Int. J. Mod. Phys. A 21, 3989 (2006). arXiv:hep-ph/0508144v1

30. Li, B.-Q., Chao, K.-T.: Phys. Rev. D 79, 094004 (2009). arXiv:0903.5506v2

31. Li, B.-Q., Chao, K.-T.: Commun. Theor. Phys. 52, 653 (2009). arXiv:0909.1369v1

32. Rai, A.K., Patel, B., Vinodkumar, P.C.: Phys. Rev. C 78, 055202 (2008). arXiv:0810.1832v1

33. Patel, S., Vinodkumar, P.C., Bhatnagar, S.: nopunct. Chin. Phys. C 40, 053102 (2016). arXiv: $1504.01103 \mathrm{v} 3$

34. Monteiro, A.P., Bhat, M., Vijaya Kumar, K.B.: Phys. Rev. D 95, 054016 (2017). arXiv:1608.05782v3

35. Kher, V., Rai, A.K.: Chin. Phys. C 42, 083101 (2018). arXiv:1805.02534v1

36. D’Souza, P.P., Monteiro, A.P., Vijaya Kumar, K.B.: Commun. Theor. Phys. 71, 192 (2019). arXiv: 1703.10413

37. Brambilla, N., Pineda, A., Soto, J., Vairo, A.: arXiv:hep-ph/0410047v2. Rev. Mod. Phys. 77, 1423 (2005)

38. Bodwin, G.T., Braaten, E., Lepage, G.P.: Phys Rev. D 51, 1125 (1995). Erratum:, Phys. Rev. D 55, 5853 (1997)

39. Guo, F.-K., Hanhart, C., Li, G., Meißner, U.G., Zhao, Q.: Phys. Rev. D 83, 034013 (2011). arXiv: $1008.3632 \mathrm{v} 2$

40. Smith, C.H.L.: Ann. Phys. 53, 521 (1969)

41. Alkofer, R., von Smekel, L.: Phys. Rep. 353, 281 (2001). arXiv:hep-ph/0007355v2

42. Maris, P., Roberts, D.: Int. J. Mod. Phys. E 12, 297 (2003). arXiv:nucl-th/0301049v1

43. Bhatnagar, S., Alemu, L.: Phys. Rev. D 97, 034021 (2018). arXiv:1610.03234v5

44. Wang, G.-L.: Phys. Lett. B 633, 492 (2006). arXiv:math-ph/0512009v1

45. Cvetic, G., Kim, C.S., Wang, G.-Li., Namgung, W.: Phys. Lett. B 596, 84 (2004)

46. Wang, Z.-G., Yang, W.-M., Wan, S.-L.: Phys. Lett. B 615, 79 (2005). arXiv:hep-ph/0411142v2

47. Negash, H., Bhatnagar, S.: Intl. J. Mod. Phys. E 25, 1650059 (2016). arXiv:1508.06131v3

48. Blank, M., Krassnigg, A.: Phys. Rev. D 84, 096014 (2011). arXiv:1109.6509v1

49. Bhagwat, M.S., Maris, P.: Phys. Rev. C 77, 025203 (2008). arXiv:nucl-th/0612069v1

50. Choi, H.-M.: Phys. Rev. D 75, 073016 (2007). arXiv:hep-ph/0701263v2

51. Mojica, F.F., Vera, C.E., Rojas, E., El-Bennich, B.: Phys. Rev. D 96, 014012 (2017). arXiv: $1704.08593 \mathrm{v} 1$

52. Bailas, G., Blossier, B., Morenas, V.: Eur. Phys. J. C 78, 1018 (2018). arXiv:1803.09673v1

53. Van Royen, R., Weisskopf, V.F.: Nuovo Cim. 50, 617 (1967). ibid. 51, 583 (1967)(E)

54. Hwang, D.S., Kim, G.-H.: Z. Phys. C 76, 107 (1997). arXiv:hep-ph/9703364v1

55. Ahmady, M.R., Mendel, R.R.: Phys. Rev. D 51, 141 (1995). arXiv:hep-ph/9401315v2

56. Edward, K.W., et al., CLEO Collaboration: Phys. Rev. Lett. 86, 30 (2001). arXiv:1010.3110v2

57. Veli Veliev, E., et al.: J. Phys. G Nucl. Part. Phys. 39, 015002 (2012). arXiv:1010.3110v2

58. Veli Veliev, E., et al.: Eur. Phys. J. A 47, 110 (2011). arXiv:1103.4330v1

59. Braaten, E., Fleming, S.: Phys. Rev. D 52, 181 (1995). arXiv:hep-ph/9501296v2

60. Kiselev, V.V.: Int. J. Mod. Phys. A 11, 3689 (1996)

61. Gershtein, S.S., et al.: arXiv:hep-ph/9803433v1 (1998)

62. Lucha, W., Schoberl, F.F.: Int. J. Mod. Phys. C 10, 607 (1999). arXiv:hep-ph/9811453v2

63. Dudek, J.J., Edwards, R.G., Richards, D.G.: Phys. Rev. D 73, 074507 (2006). arXiv:hep-ph/0601137v2

64. Davies, C.T.H., et al., HPQCD collaboration: Phys. Rev. D 82, 114504 (2010). arXiv:1008.4018v2

65. Donald, G.C., et al., HPQCD collaboration: Phys. Rev. D 86, 094501 (2012). arXiv:1208.2855v2

66. McNeile, C., et al., HPQCD collaboration: Phys. Rev. D 86, 074503 (2012). arXiv: 1207.0994v1

67. Colquhoun, B., et al., HPQCD collaboration: Phys. Rev. D 91, 074514 (2015). arXiv:1408.5768v1

68. Chiu, T.W., et al., TWQCD Collaboration: Phys. Lett. B 651, 171 (2007). arXiv:0705.2797v1

69. Soni, N.R., Joshi, B.R., Shah, R.P., Chauhan, H.R., Pandya, J.N.: Eur. Phys. J. C 78, 592 (2018). arXiv: $1707.07144 \mathrm{v} 2$

Publisher's Note Springer Nature remains neutral with regard to jurisdictional claims in published maps and institutional affiliations. 